\title{
Cut-off Points of Treatment Delay to Predict Poor Outcomes Among New Pulmonary Tuberculosis Cases in Dalian, China: A Cohort Study
}

\author{
Haoqiang Ji $\mathbb{I}^{\prime}$ \\ Jia Xu' \\ Ruiheng Wu (iD) \\ Xu Chen' \\ Xintong $L v^{2}$ \\ Hongyu Liu ${ }^{2}$ \\ Yuxin Duan' \\ Meng Sun' \\ Yuanping Pan' \\ Yunting Chen' \\ Xiwei $\mathrm{Lu}^{2}$ \\ Ling Zhou'
}

'School of Public Health, Dalian Medical University, Dalian, I I6044, People's Republic of China; ${ }^{2}$ Office of Epidemic Surveillance, Dalian Tuberculosis Hospital, Dalian, Liaoning, People's Republic of China
Correspondence: Xiwei Lu; Ling Zhou 9 West Section, Lvshun South Road, Dalian, Liaoning Province, People's Republic of China

Tel +864II 86II 0368

Email yiluxiwei@126.com;

zhouling0609@I63.com
Purpose: Despite increasing literature on the association between treatment delay and outcomes, cut-off point ( 1 month or median) selection in almost all studies for treatment delay is too subjective. This study explored more scientific cut-off points of treatment delay for poor treatment outcomes and death at the clinical level.

Patients and Methods: A total of 18,100 newly confirmed pulmonary tuberculosis (TB) cases in Dalian, China were used in the final analysis. A 3-knotted restricted cubic spline (RCS) fitted for Cox proportional hazard regression models is used to analyse the effects of cut-off points of treatment delay on incident poor treatment outcomes. To explore the moderating effects of age, gender and diabetes, we added the interaction terms of these moderating variables and treatment delay to Cox proportional hazard regression models.

Results: The median time of treatment initiation was 30 days (IQR: 14-59 days). The risk of incident poor treatment outcomes increased when the time was greater than cut-off point 1 (53 days; adjusted HR: 1.26; 95\% CI: 1.00-1.60) of treatment delay, and the risk of incident death events increased when the time was greater than cut-off point 2 (103 days; adjusted HR: 1.56 ; 95\% CI: 1.00-2.44) of delay. In addition, treatment delay was associated with an increased risk of incident poor treatment outcomes and death, and older age, male sex, and diabetes may increase the risk of treatment delay for poor outcomes.

Conclusion: This study is the first to identify scientific cut-off points of treatment delay for poor treatment outcomes and death, and this method of exploration should be popularized. In addition, the knowledge of tuberculosis must be spread to every adult. Moreover, the tuberculosis diagnosis level of community level health workers should be enhanced.

Keywords: new pulmonary tuberculosis patients, poor treatment outcomes, treatment delay, cut-off points, restricted cubic spline

\section{Introduction}

Tuberculosis (TB) is, caused by Mycobacterium tuberculosis. TB is, a communicable disease that is a major cause of ill health, one of the top 10 causes of death worldwide and the leading cause of death from a single infectious agent (ranking above HIV/ AIDS). ${ }^{1,2}$ According to the World Health Organization (WHO) Global Tuberculosis Report 2021, an estimated 9.9 million (incidence rate: 127 per 100,000) individuals were diagnosed with TB with 1.5 million deaths in $2020 .^{3}$ Although these indicators are declining slowly each year, the pace of the decline in many countries was too slow to reach the 2020 milestones of the End TB Strategy. ${ }^{2,4}$ China is also one of those countries, and the second-highest TB burden country (accounting for approximately 
$9 \%$ of cases following India) worldwide even though the country provides some free anti-TB medicines and subsidies to patients. ${ }^{5}$ In China, approximately 833,000 (range: 717,000-957,000) new TB cases were estimated in 2019, accounting for approximately $8.4 \%$ of total newly confirmed TB cases worldwide, ${ }^{2}$ ranking third in the world cases. In addition, $7.1 \%$ of new cases in China were MDR/RR-TB, and DR-TB accounted for $14 \%$ of the world cases, ranking second. ${ }^{2}$ Therefore, many new patients and existing cases will add a heavy burden to Chinese TB prevention and control efforts.

Antibiotic treatment is the most important component of the End TB Strategy, and early diagnosis and treatment are effective in the TB control program. ${ }^{6}$ However, many newly diagnosed TB cases face not only financial difficulties but also a lack of poor awareness about TB prevention and treatment. ${ }^{7,8}$ For example, two surveys showed that only $16 \%$ and $26.9 \%$ of adults had accurate knowledge of TB symptoms in two Chinese provinces, ${ }^{9,10}$ and another survey showed that the total mean percentage of correct answers for TB diagnosis knowledge was $35.7 \%$ (SD: 16.1\%) among medical students in Hunan, China. ${ }^{11}$ Many people do not have sufficient knowledge to recognize TB and treat TB as a cold or pneumonia and initially go to drugstores or village clinics to buy cold medicines or antibiotics to relieve the disease when they develop TB symptoms. ${ }^{9,12}$ In addition, there is also a lack of TB knowledge in the health system. ${ }^{13}$ The clinical features of TB cases are similar to those of other chest diseases, and health care workers at the community level may not be able to diagnose TB in a timely manner. ${ }^{14,15}$ One survey showed that $84.7 \%$ of new TB cases were diagnosed after an average of 3.6 misdiagnoses (SD: 2.4) in Ethiopia. ${ }^{12}$ This problem seems to exist in all countries with a systematic review of studies in 78 countries showing that the median time of treatment initiation for total TB cases was 88 days (range: $21-136$ days). ${ }^{16}$ During the COVID-19 pandemic, newly treated TB patients face additional barriers to seeking health care, so many patients go undiagnosed, and a long time has passed before starting treatment. ${ }^{17}$ If these patients are unfortunate enough to contract COVID-19 while seeking care, this will undoubtedly worsen their illness. ${ }^{18}$ Blindly seeking and delaying treatment not only lead to antibiotic overuse but also may delay the start of effective treatment and cause TB transmission in the community. ${ }^{19,20}$ When patients miss the optimal time for treatment, this will lead to a series of serious consequences, ${ }^{21}$ such as disease progression, ${ }^{22}$ drug resistance and poor outcomes. ${ }^{23}$ Although many worldwide studies on the correlation between treatment delay and outcomes have increased gradually, no study has identified a standard cut-off point of treatment delay or the best time of treatment initiation to yield good outcomes. ${ }^{12}$

To our knowledge, almost all these studies used 1 month or the median time of treatment initiation as cut-off points. ${ }^{24,25}$ These points were highly subjective, and some important information will be lost when the data are converted into categorical variables. More importantly, without an accurate cut-off point, patients may ignore symptoms or blindly seek treatment. ${ }^{26}$ Many Chinese people have the bad habit of procrastinating when they get sick, because they think they are healthy enough to fight it off or completing what they were initially doing first. Therefore, it is significant to explore an accurate cut-off point for clinical treatment and academic research. Under these circumstances, we used a 3-knotted restricted cubic spline fitted for Cox proportional hazard regression models to explore the cut-off point for poor treatment outcomes and the relationship between the time of treatment initiation and the risk of incident poor outcomes in Dalian, China.

\section{Materials and Methods Study Population}

Dalian, which is located in northeast China, has a residential population of 6.95 million, and suffers from a high TB burden. ${ }^{27}$ The incidence of TB was 52.8 per 100,000 , and new MDR/RR-TB cases accounted for $4.8 \%$ in the region, higher than the global level. ${ }^{27}$ In Dalian, information from all TB patients is collected regularly with TB surveillance and management through the China Information System for TB Prevention and Control. TB cases in the study were diagnosed by sputum smear examination, sputum culture or the WHO clinical criteria from Jan 1, 2014, to Dec 31, 2019. A total of 21,810 TB patients were treated and registered in Dalian, China. Considering that minors (less than 18 years old) and persons with mental illness lack the thinking and behaviour of normal adults, and have unique legal status in China, they cannot correctly identify their own behaviour during diagnosis and treatment, affecting the scientific results. Therefore, minors and mental patients were excluded from this study. In addition, we excluded patients with extrapulmonary TB and relapse cases according to the criteria of the TB cohort study $^{28}$ and eventually included 18,100 newly diagnosed pulmonary TB cases. Figure 1 shows the details of case inclusion and exclusion. 




Figure I The flow chart of the inclusion of new pulmonary TB cases.

\section{Treatment Outcomes}

All newly confirmed TB patients typically require at least 6 months of standard therapy (WHO protocol for drug use). ${ }^{28}$ In addition, patients cannot continue to use standard therapy due to drug resistance, adverse reactions, etc., whereas new treatment regimens developed after expert discussion are defined as experiential therapy. At the end of the treatment, doctors registered each patient's treatment outcomes. The primary treatment outcomes in this study were WHO-defined TB treatment outcomes: ${ }^{28}$ cure, treatment completion, failure, default and death. Cure or treatment completion was defined as favoured outcomes. Failure, default and death were defined as poor outcomes.

\section{Time of Treatment Initiation}

The time of treatment initiation was assessed by asking patients to recall the date or number of days that elapsed between anti-TB treatment initiation and the onset of TB symptoms, such as cough, fever, night sweats, chest pain, and weight loss. In addition, due to lack of a standard cut-off point and to first explore the association between outcomes and the change in time, we divided the time into four groups (quartile 1-quartile 4; Q1: 0-14 days; Q2: 15-30 days; Q3: 3159 days; Q4: 60 days and above) at the quartile cut-off point to represent an ordered change in initiation treatment time. 


\section{Covariates}

We adjusted for 3 types of potential covariates, including demographic characteristics, health status and treatment regimens, in multivariate analysis. The demographic characteristics included gender (male/female), age (young:1844 years/middle:45-64 years/elderly: 65 years and above), resident area (urban/rural area), and occupation (employed/unemployed). Health status included diabetes mellitus (Yes/ No), drug resistance (judging by drug susceptibility testing; Yes/No), severe case (Yes/No), lung cavitation (Yes/No), and treatment options (standard therapy/experiential therapy).

\section{Statistical Analysis}

Data were filtered and analysed using Stata 14.0. The characteristics of new TB cases were described as percentages (\%) for categorical variables or medians and interquartile ranges (IQRs) for continuous variables. Wilcoxon rank-sum tests or Kruskal-Wallis tests were used to compare group differences in the time of treatment initiation. Second, to explore the association between treatment outcomes and the time change of treatment initiation, we first added categorical variables (divided by quartile cut-off points) of initiation time to Cox proportional hazard regression models adjusted for all covariates (age, gender, resident area, occupation, drug resistance, severe case, treatment options and lung cavitation). Third, as a method to analyse the dose response relationship, the RCS model can describe the curve relationship between continuous independent variables and outcome in more detail, instead of grouping the independent variables, which can reduce the loss of independent variable information. After adjusting for all covariates, we used 3-knotted RCS fitted for Cox proportional hazard regression models to explore the cut-off point of treatment delay and the associations between changes in delay time and poor outcomes. Fourth, to explore the moderating effects of age, gender and diabetes, we added interactions of moderating variables and treatment delay to the Cox proportional hazard models, and used the adjusted hazard ratio (AHR) with $95 \%$ confidence intervals (CIs) to show these regression results. Two-sided $\mathrm{P}<0.05$ was defined as statistically significant. All analyses were performed using Stata version 14.0 (StataCorp) and $\mathrm{R}$ version 4.1.0 (R Foundation).

\section{Ethical Considerations}

The study was approved by the Ethics Committee of Dalian Medical University and Dalian Tuberculosis Hospital, Dalian, China. We obtained permission from the hospital administrators to use the data. Information on this study was anonymized and personal identifiers were not included. The design and protocol of this study completely considered the principles of safety and security, and was conducted in accordance with the Declaration of Helsinki.

\section{Results \\ Characteristics}

A total of 18,100 new TB cases were included in the final analyses, and the median time of treatment initiation was 30 days (IQR: 14-59 days). The median time between symptom onset and the first medical visit was 21 days (IQR: 7-46), and $96.1 \%$ of patients started treatment on the day of diagnosis. Moreover, the following factors were associated with a longer median time of treatment initiation: older age, female, rural, employed, drug resistant, diabetes, lung cavitation and undergoing experiential therapy ( $\mathrm{P}<0.05$ for all) (Table 1).

\section{Risk Factors for Poor Treatment Outcomes}

In multivariate Cox regression models, compared with references (Q1), Q4 (60 days and above) was a risk factor for poor treatment outcomes (AHR: 1.43; 95\% CI: 1.15 1.78). In addition, when death was defined as an outcome event, Q4 (60 days and above) (AHR: 1.49, 95\% CI: 1.052.13) was associated with death. Table 2 shows details of the risk factors for incident poor outcomes.

\section{The Cut-off Points of Treatment Delay for Poor Outcomes}

In 3-knotted restricted cubic spline fitted for Cox regression models, when treatment was delayed for more than 53 days (AHR: $1.26 ; 95 \%$ CI: $1.00-1.60$ ), the risk of incident poor treatment outcomes increased gradually and statistically significant. When death was considered an outcome event and the time was more than 103 days (AHR: $1.56 ; 95 \%$ CI: 1.00-2.44), the risk of incident death events increased gradually. Figure 2 shows the trend of the risk of incident outcome events with time of delay treatment. 
Table I Characteristics of New Pulmonary TB Cases Base on the Time of Treatment Initiation

\begin{tabular}{|c|c|c|c|c|}
\hline Characteristics & $\mathbf{N}(\%)$ & Time of Treatment Initiation, M (IQR) & $\mathbf{z}$ & $P$ value \\
\hline Total & $18,100(100)$ & $30(14-59)$ & & \\
\hline Age & & & 529.779 & $<0.001$ \\
\hline Young & $9063(50.1)$ & $25(\mid 2-44)$ & & \\
\hline Middle & $6170(34.1)$ & $32(17-66)$ & & \\
\hline Elderly & $2867(15.9)$ & $34(20-70)$ & & \\
\hline Gender & & & 11.426 & $<0.001$ \\
\hline Female & $5747(31.8)$ & $31(15-62)$ & & \\
\hline Male & $12,353(68.2)$ & $30(14-57)$ & & \\
\hline Residence & & & 116.402 & $<0.001$ \\
\hline Rural & $10,293(56.9)$ & $31(15-64)$ & & \\
\hline Urban & $7807(43.1)$ & $29(14-46)$ & & \\
\hline Employment status & & & 38.357 & $<0.001$ \\
\hline Employed & $6989(38.6)$ & $31(15-64)$ & & \\
\hline Unemployed & $I I, I I \mid(6 I .4)$ & $30(\mid 4-53)$ & & \\
\hline Drug resistance & & & 16.063 & $<0.001$ \\
\hline Yes & $563(3.1)$ & $32(18-68)$ & & \\
\hline No & $17,537(96.9)$ & $30(14-59)$ & & \\
\hline Diabetes mellitus & & & $17.16 \mid$ & $<0.001$ \\
\hline Yes & $897(5)$ & $31(16-66)$ & & \\
\hline No & $17,203(95)$ & $30(14-59)$ & & \\
\hline Severe case & & & 0.346 & 0.557 \\
\hline Yes & $1709(9.4)$ & $3 I(\mid 4-6 I)$ & & \\
\hline No & $|6,39|(90.6)$ & $30(14-59)$ & & \\
\hline Lung cavitation & & & 130.066 & $<0.001$ \\
\hline Yes & $3800(21)$ & $34(|6-7|)$ & & \\
\hline No & $14,305(79)$ & $29(14-53)$ & & \\
\hline Treatment options & & & 25.823 & $<0.001$ \\
\hline Standard therapy & $17,247(95.3)$ & $30(14-59)$ & & \\
\hline Experiential therapy & $853(4.7)$ & $32(19-61)$ & & \\
\hline
\end{tabular}

Abbreviations: $M$, median; IQR, interquartile range.

\section{Moderating Effects of Age, Gender, Diabetes Mellitus}

When 53 days and 103 days were defined as the cut-off point of incident poor outcomes and death, the moderating effects of age, sex, and diabetes mellitus were significant. Compared with the references, older patients (45 years and above) with delayed treatment had a higher risk of incident poor treatment outcomes (AHR: 3.17, 95\% CI: 2.51-4.02) and death (AHR: 11.38, 95\% CI: 6.65 19.46). In addition, male patients with treatment delay had a higher risk of poor treatment outcomes (AHR: 2.30, 95\% CI: 1.74-3.04) and death (AHR: 2.52, 95\% CI: 1.60-3.98). Moreover, patients with diabetes mellitus who received delayed treatment had a higher risk of incident poor treatment outcomes (AHR: 1.50, 95\% CI: 1.00-2.25) (Figure 3).

\section{Discussion}

This study is the first to focus on the relationship between the time of treatment initiation and treatment outcomes in northeast China and explore cut-off points of treatment delay. The study found that when the time of treatment initiation was greater than 53 days and 103 days, the risk of incident poor outcomes and death events increased gradually using 3-knotted restricted cubic spline (RCS) fitted for Cox proportional hazard regression models. These cut-off points of treatment delay are more scientifically grounded than the median of the time or 1 month at clinical treatment level, and should be used as TB treatment evidence to educate people in Dalian, China. The method and experience should also be used to explore the cut-off point in other countries with a high TB burden, because some countries still face a higher rate of treatment 
Table 2 The Association Between Incident Poor Outcomes and Risk Factors in Cox Regression Models

\begin{tabular}{|c|c|c|c|c|c|c|}
\hline \multirow[t]{2}{*}{ Independent Variables } & \multicolumn{3}{|c|}{ Poor Treatment Outcomes } & \multicolumn{3}{|l|}{ Death } \\
\hline & $\begin{array}{l}\text { Incidence Rate, } \\
\text { Per } 1000\end{array}$ & AHR & $95 \% \mathrm{Cl}$ & $\begin{array}{l}\text { Incidence Rate, } \\
\text { Per } 1000\end{array}$ & AHR & $95 \% \mathrm{Cl}$ \\
\hline \multicolumn{7}{|c|}{ Time of treatment initiation } \\
\hline QI (0-14 days) & 28.3 & Ref. & Ref. & 9.8 & Ref. & Ref. \\
\hline Q2 (15-30 days) & 34.1 & 1.07 & $0.85-1.35$ & 12.5 & 0.99 & $0.67-1.47$ \\
\hline Q3 (3I-59 days) & 39.9 & 1.15 & $0.91-1.44$ & 13.3 & 0.95 & $0.64-1.41$ \\
\hline Q4 (60 days and above) & 54.3 & $\mathrm{I} .43$ & I.I5-I.78 & 22.6 & 1.49 & $1.05-2.13$ \\
\hline \multicolumn{7}{|l|}{ Gender } \\
\hline Female & 21.4 & Ref. & Ref. & 7.7 & Ref. & Ref. \\
\hline Male & 47.3 & 1.78 & $1.46-2.17$ & 17.7 & 1.94 & $1.40-2.69$ \\
\hline \multicolumn{7}{|l|}{ Age } \\
\hline Young & 19.6 & Ref. & Ref. & 3.0 & Ref. & Ref. \\
\hline Middle & 47.5 & 1.85 & I.53-2.24 & 12.6 & 3.59 & $2.30-5.59$ \\
\hline Elderly & 82.3 & 3.57 & $2.92-4.36$ & 55.1 & 15.64 & $|0.32-23.7|$ \\
\hline \multicolumn{7}{|l|}{ Residence } \\
\hline Rural & 37 & Ref. & Ref. & 15.4 & Ref. & Ref. \\
\hline Urban & 41.8 & 1.37 & $1.15-1.63$ & 13.4 & 1.07 & $0.80-1.43$ \\
\hline \multicolumn{7}{|l|}{ Employment status } \\
\hline Unemployed & 35.5 & Ref. & Ref. & 13.3 & Ref. & Ref. \\
\hline Employed & 41.3 & 1.21 & $1.02-1.45$ & 15.3 & 1.25 & $0.93-1.68$ \\
\hline \multicolumn{7}{|l|}{ Drug resistance } \\
\hline No & 32 & Ref. & Ref. & 13.6 & Ref. & Ref. \\
\hline Yes & 259.3 & 6.98 & $5.76-8.44$ & 42.6 & 2.72 & $1.77-4.17$ \\
\hline \multicolumn{7}{|l|}{ Treatment options } \\
\hline Standard therapy & 37.2 & Ref. & Ref. & 13.1 & Ref. & Ref. \\
\hline Experiential therapy & 76.2 & 1.34 & $1.03-1.74$ & 43.4 & 1.93 & I.35-2.75 \\
\hline \multicolumn{7}{|l|}{ Severe case } \\
\hline No & 38.1 & Ref. & Ref. & 14.9 & Ref. & Ref. \\
\hline Yes & 48 & 0.93 & $0.72-1.21$ & II.I & 0.66 & $0.39-1.11$ \\
\hline \multicolumn{7}{|l|}{ Diabetes mellitus } \\
\hline No & 36.6 & Ref. & Ref. & 14.0 & Ref. & Ref. \\
\hline Yes & 87 & 1.28 & $0.99-1.65$ & 24.5 & 0.98 & $0.62-1.56$ \\
\hline \multicolumn{7}{|l|}{ Lung cavitation } \\
\hline No & 30 & Ref. & Ref. & 13.4 & Ref. & Ref. \\
\hline Yes & 73.2 & 1.91 & $1.60-2.28$ & 18.7 & 1.36 & $1.00-1.85$ \\
\hline
\end{tabular}

Abbreviations: AHR, adjusted hazard ratio; $\mathrm{Cl}$, confidence interval; Q, quartile; Ref, reference;

failure and more serious problems of treatment delay compared with China. A clear cut-off point of treatment delay can increase the urgency for patients to seek medical care and attract the attention of local governments. In addition, we found that treatment delay has negative effects on incident poor outcomes and death events in Dalian. Finally, new cases with delayed treatment who were male, older and diabetic will have an increased risk of incident poor treatment outcomes compared with their references.

Since the Directly Observed Treatment short course strategy (DOTs) was implemented in Dalian, the quality and completion of treatment have improved for TB cases, but the problem of treatment delay has not received equal attention. The study found that the median time for patients to start treatment was 30 days (IQR: 14-59 days), and $96.1 \%$ of patients started treatment on the day of diagnosis, demonstrating that patients are highly motivated to start treatment. However, it takes a long time for patients to seek the first care, which may be reflected poor awareness of TB. Therefore, educating the general population about TB symptoms and the dangers of delayed treatment is a primary concern to address the problem of treatment delay. After reviewing the extensive literature, 

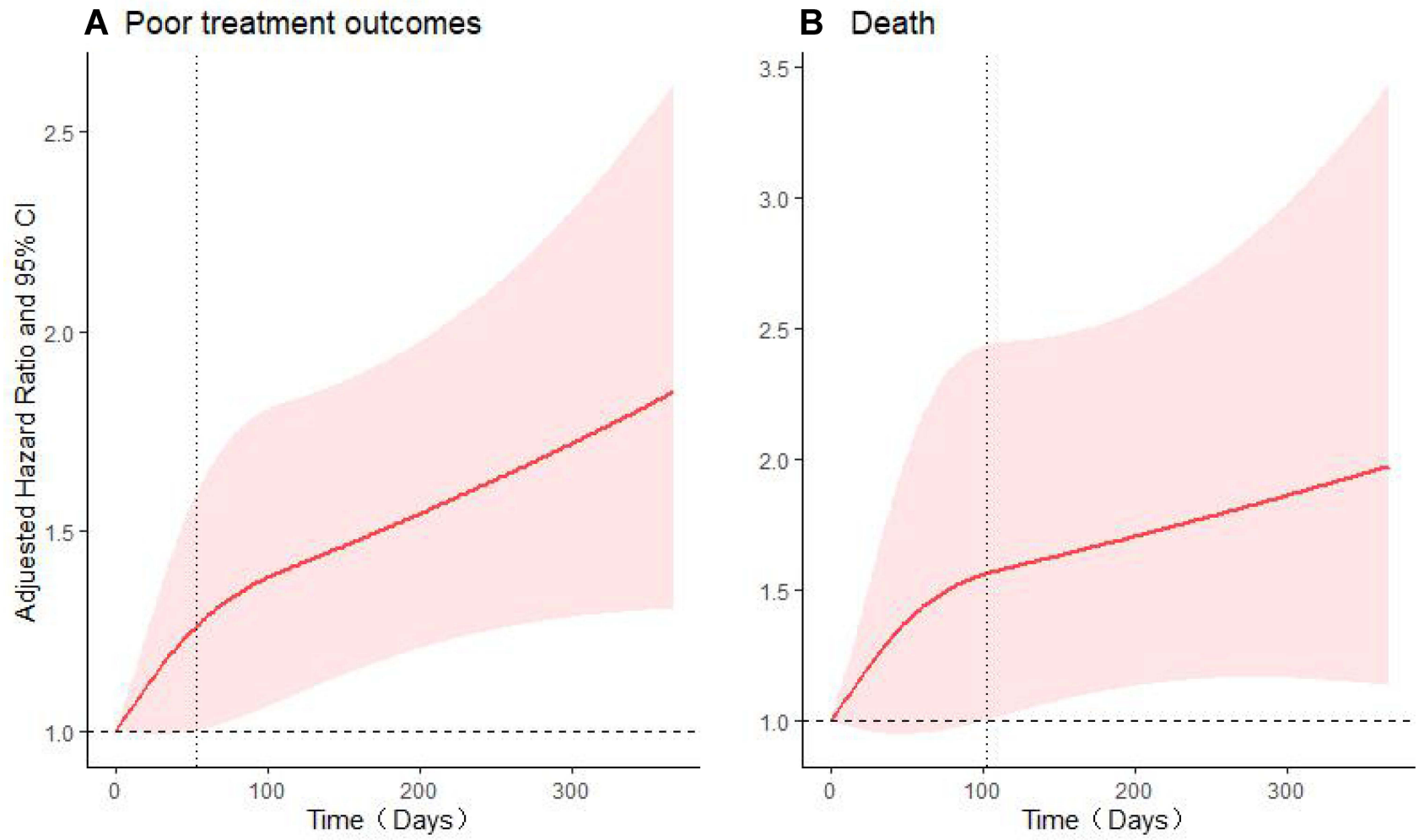

Figure 2 Adjusted hazard ratios (AHRs) of treatment outcomes event risk according to the time of treatment initiation.

Notes: In RCS fitted for Cox regression models, we adjusted for age, gender, resident area, occupation, drug resistance, severe case, lung cavitation, treatment options and lung cavitation.

Independent variables
Non treatment delay
Treatment delay
Treatment delay (Age)
Non delay (young cases)
Non delay (Older cases)
Delay (young cases)
Delay (Older cases)
Treatment delay (Gender)
Non delay (Female cases)
Non delay (Male cases)
Delay (Female cases)
Delay (Male cases)
Treatment delay (Diabetes)
Non delay (No diabetes)
Non delay (Diabetes)
Delay (No diabetes)
Delay (Diabetes)

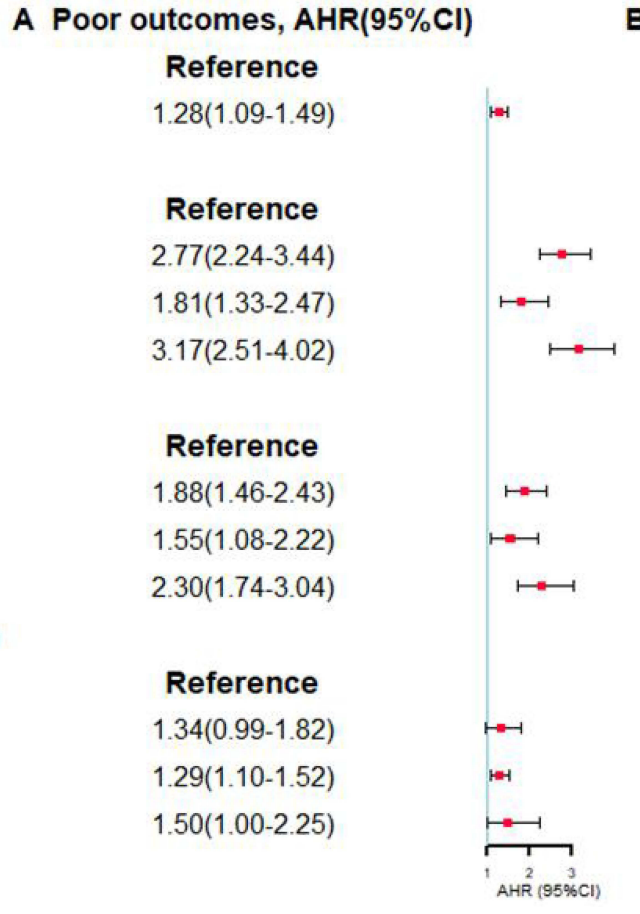

B Death, AHR( $95 \% \mathrm{Cl})$

\section{Reference}

1.38(1.01-1.89)

Reference

9.14(5.75-14.52)

$3.64(1.54-8.62)$

$11.38(6.65-19.46)$

\section{Reference}

$1.76(1.22-2.52)$

$1.16(0.54-2.49)$

$2.52(1.60-3.98)$

Reference

$0.99(0.60-1.64)$

1.37(0.99-1.89)

$1.30(0.48-3.54)$

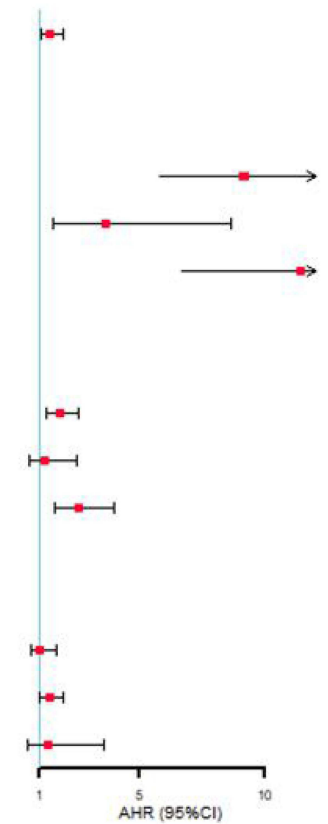

Figure 3 The moderating effects of age, gender and diabetes mellitus.

Notes: To assess the moderating effects of age, gender, and diabetes mellitus, we adjusted for age, gender, resident area, occupation, drug resistance, severe case, lung cavitation, treatment options and lung cavitation in Cox regression models. 
the problem of patient delay exists in almost all countries with a TB high burden, but the problem seems to be worse in developing countries. ${ }^{25,29,30}$ This finding is related to the TB knowledge of citizens and the diagnostic level of primary medical institutions, ${ }^{31,32}$ which may make TB control more difficult to achieve in poor countries. In addition, TB notification and timely treatment are also are impacted by the COVID-19 pandemic. According to a study from Ningxia, China, the TB notification rates decreased by greater than $60 \%$ in the COVID- 19 pandemic compared with the average level. The delay in treatment during this period was significantly longer than that noted before the pandemic. ${ }^{33}$ The COVID-19 pandemic has resulted in more patients not receiving timely and effective treatment, resulting in TB community spread and increasing the burden of TB control. ${ }^{2}$

Under these circumstances, those with undetected TB will continue to experience worsening symptoms and take longer to start treatment, adding to difficulties of TB treatment. Old TB cases with treatment delay have an increased risk of incident poor outcomes. In older patients, their physical condition worsens, and these patients have some age-related diseases, such as chronic comorbidities and dysfunction in cellular immunity. ${ }^{34}$ Therefore, the clinical presentations of TB can be easily confused with other agerelated illnesses, ${ }^{35}$ and these patients are more prone to misdiagnosis and experience adverse events, such as severe hepatotoxicity during anti-TB treatment. ${ }^{36}$ More attention should be given to this vulnerable group, and earlier treatment may be the best choice for these older patients. ${ }^{37}$ In addition, male TB cases who delay treatment have a higher risk of incident poor outcomes. Men are often the main breadwinners of a family in relatively backwards areas, and under more family responsibilities and pressure. Once these males are hospitalized or seek treatment in a TB hospital in a remote location, the process may take so long that it affects their income. Therefore, they often choose a nearby pharmacy to buy medication for their symptoms and have longer time of treatment initiation. ${ }^{38}$ In addition, men tend to have bad living habits such as smoking, drinking and forgetting to take medicines, which increase the difficulty of TB treatment. Moreover, TB patients with diabetes and delayed treatment have a high risk of treatment failure. One study showed that diabetic patients are not only more susceptible to TB infection but also have higher mycobacterial burdens at the beginning of treatment and treatment difficulty. ${ }^{39}$ Other studies found that alveolar macrophages of diabetic patients were less activated and exhibited decreased hydrogen peroxide production, which are favourable conditions for Mycobacterium tuberculosis. Thus, diabetic patients seem to need a longer time to achieve a negative sputum culture. ${ }^{40,41}$ Additionally, the physical condition of TB cases with diabetes mellitus is very poor, and the efficacy of TB treatments are reduced when combined with hypoglycaemic drugs and antibiotics. ${ }^{42}$ Therefore, the use of predictive host biomarkers and tailored anti-TB therapy in patients with diabetes should be developed to solve this problem and thus improve treatment effectiveness. ${ }^{43}$

Overall, male, middle-aged and elderly and diabetic patients, which represent vulnerable groups in TB treatment, must receive timely attention. Global strategies and national TB programmes should identify these groups as underserved higher-risk groups and improve their access to diagnosis and screening services. ${ }^{38}$ Primary health care institutions are the first choice for many patients, so the ability of primary medical staff to diagnose TB must be strengthened. However, this problem represents a significant challenge in China. More importantly, governments and health care officers must spread knowledge of TB (TB symptoms, treatment delay, etc.) to every person and include these cut-off points in the TB control manual given that this lack of knowledge is a risk of poor outcomes. When people understand the risk of treatment delay for poor outcomes, they attach greater importance to TB and TB-related symptoms, and increase the urgency of seeking medical care once symptoms appear.

\section{Limitations}

Several limitations should be mentioned in this study. First, the calculation of cut-off points only takes into account the patient's treatment outcomes, not the infectivity of TB. Under the circumstance of the End TB Strategy, the earlier treatment starts, the more it prevents the spread of TB. Second, the results are only applicable to new pulmonary TB in Dalian, China. However, this cut-off point could be used as a methodology for other treatment delay studies to explore the difference in treatment delay in other countries or regions, and a further analysis of the reasons behind these differences may be highly informative for control policies. Third, the time of treatment initiation was assessed based on the subjective memory of cases, which may result in recall bias. However, we used local and national events to minimize this bias. Fourth, some important confounding factors, such as smoking status, drink status, and treatment adherence, were not included. 


\section{Conclusion}

This study is the first to identify more scientific cut-off points of treatment delay to predict poor outcomes using RCS regression. When the time of treatment initiation is longer than the cut-off point (53 days), the risk of incident poor treatment outcomes increases significantly. For academic research and optimization of clinical treatment, cutoff points or this method should be promoted, and concern arises. In addition, treatment delay is a risk factor for incident poor treatment outcomes and death events. New TB patients with treatment delay who were male, older, diabetic had an increased risk of incident poor treatment outcomes than their references. Policies to increase TB knowledge must be implemented.

\section{Abbreviations}

TB, tuberculosis; WHO, World Health Organization; $\mathrm{SD}$, standard deviation; M, median; IQR, interquartile range; Q, quartile; RCS, restricted cubic spline; AHR, adjusted hazard ratio; $\mathrm{CI}$, confidence interval; Ref, reference; DOTs, directly observed treatment short course strategy.

\section{Acknowledgments}

We would like to express our deep appreciation to all of the individuals who contributed to this study. We are grateful to the staff of Dalian Tuberculosis Hospital who contributed to this study.

\section{Author Contributions}

$\mathrm{HJ}$ contribution to conception and design, analysis, interpretation of data and drafting the article. All authors made substantial contributions to acquisition of data, revising it critically for important intellectual content; agreed to submit to the current journal; gave final approval of the version to be published; and agree to be accountable for all aspects of the work.

\section{Funding}

The authors have not declared a specific grant for this research from any funding agency in the public, commercial or not-for-profit sectors.

\section{Disclosure}

The authors report no conflicts of interest in this work.

\section{References}

1. Furin J, Cox H, Pai M. Tuberculosis. Lancet. 2019;393 (10181):1642-1656. doi:10.1016/s0140-6736(19)30308-3

2. World Health Organization. Global Tuberculosis Report 2020. Geneva, Switzerland; 2020.

3. World Health Organization. Global Tuberculosis Report 2021. Geneva, Switzerland; 2021.

4. Floyd K, Glaziou P, Zumla A, Raviglione M. The global tuberculosis epidemic and progress in care, prevention, and research: an overview in year 3 of the End TB era. Lancet Resp Med. 2018;6(4):299-314. doi:10.1016/s2213-2600(18)30057-2

5. Wang LX, Zhang H, Ruan YZ, et al. Tuberculosis prevalence in China, 1990-2010; a longitudinal analysis of national survey data. Lancet. 2014;383(9934):2057-2064. doi:10.1016/s0140-6736(13) 62639-2

6. Storla DG, Yimer S, Bjune GA. Can treatment delay be utilized as a key variable for monitoring the pool of infectious tuberculosis in a population? J Infect Dev Ctries. 2010;4(2):83-90. doi:10.3855/ jidc. 211

7. Teo AKJ, Singh SR, Prem K, Hsu LY, Yi SY. Duration and determinants of delayed tuberculosis diagnosis and treatment in high-burden countries: a mixed-methods systematic review and meta-analysis. Respir Res. 2021;22(1):251. doi:10.1186/s12931-021-01841-6

8. French CE, Kruijshaar ME, Jones JA, Abubakar I. The influence of socio-economic deprivation on tuberculosis treatment delays in England, 2000-2005. Epidemiol Infect. 2009;137(4):591-596. doi:10.1017/s0950268808001118

9. Wang JM, Fei Y, Shen HB, Xu B. Gender difference in knowledge of tuberculosis and associated health-care seeking behaviors: a cross-sectional study in a rural area of China. BMC Public Health. 2008;8354. doi:10.1186/1471-2458-8-354

10. Ma EB, Ren LP, Wang WS, et al. Demographic and socioeconomic disparity in knowledge about tuberculosis in inner Mongolia, China J Epidemiol. 2015;25(4):312-320. doi:10.2188/jea.JE20140033

11. Ou YJ, Luo ZZ, Mou JS, et al. Knowledge and determinants regarding tuberculosis among medical students in Hunan, China: a cross-sectional study. BMC Public Health. 2018:18730. doi:10.1186/s12889-018-5636-x.

12. Asres A, Jerene D, Deressa W. Delays to treatment initiation is associated with tuberculosis treatment outcomes among patients on directly observed treatment short course in Southwest Ethiopia: a follow-up study. Article. BMC Pulm Med. 2018;18:11.64. doi:10.1186/s12890-018-0628-2

13. Riccardi N, Alagna R, Motta I, et al. Towards ending TB: civil community engagement in a rural area of Senegal: results, challenges and future proposal. Infect Dis. 2019;51(5):392-394. doi:10.1080/ 23744235.2019.1572920

14. Chen CC, Chiang PH, Chen YH, Fan IC, Chan TC. Patient and health care system characteristics are associated with delayed treatment of tuberculosis cases in Taiwan. BMC Health Serv Res. 2019;19(1):846. doi:10.1186/s12913-019-4702-0

15. Getnet F, Demissie M, Assefa N, Mengistie B, Worku A. Delay in diagnosis of pulmonary tuberculosis in low-and middle-income settings: systematic review and meta-analysis. BMC Pulm Med. 2017;17202. doi:10.1186/s12890-017-0551-y

16. Storla DG, Yimer S, Bjune GA. A systematic review of delay in the diagnosis and treatment of tuberculosis. BMC Public Health. 2008;815. doi:10.1186/1471-2458-8-15

17. Migliori GB, Thong PM, Alffenaar JW, et al. Gauging the impact of the COVID-19 pandemic on tuberculosis services: a global study. Eur Respir J. 2021;58(5):2101786. doi:10.1183/13993003.01786-2021

18. Casco N, Jorge AL, Palmero DJ, et al. Tuberculosis and COVID-19 co-infection: description of the global cohort. Eur Respir J. 2021. doi:10.1183/13993003.02538-2021 
19. Golub JE, Bur S, Cronin WA, et al. Delayed tuberculosis diagnosis and tuberculosis transmission. Int J Tuberc Lung Dis. 2006;10 (1):24-30.

20. Xu L, Chongsuvivatwong V, Lu L, Geater A, Ren LJ. Dose-response relationship between treatment delay of smear-positive tuberculosis patients and intra-household transmission: a cross-sectional study. Trans Roy Soc Trop Med Hyg. 2008;102(8):797-804. doi:10.1016/j. trstmh.2008.04.027

21. Tedla K, Medhin G, Berhe G, Mulugeta A, Berhe N. Delay in treatment initiation and its association with clinical severity and infectiousness among new adult pulmonary tuberculosis patients in Tigray, northern Ethiopia. BMC Infect Dis. 2020;20(1). doi:10.1186/ s12879-020-05191-4

22. Virenfeldt J, Rudolf F, Camara C, et al. Treatment delay affects clinical severity of tuberculosis: a longitudinal cohort study. Article. BMJ Open. 2014;4(6):8.e004818. doi:10.1136/bmjopen-2014-004818

23. Ngabonziza JCS, Habimana YM, Decroo T, et al. Reduction of diagnostic and treatment delays reduces rifampicin-resistant tuberculosis mortality in Rwanda. Int J Tuberc Lung Dis. 2020;24(3):329-+. doi:10.5588/ijtld.19.0298

24. Gebreegziabher SB, Bjune GA, Yimer SA. Total delay is associated with unfavorable treatment outcome among pulmonary tuberculosis patients in West Gojjam Zone, Northwest Ethiopia: a Prospective Cohort Study. PLoS One. 2016;11(7):e0159579. doi:10.1371/journal.pone.0159579

25. Teo AKJ, Ork C, Eng S, et al. Determinants of delayed diagnosis and treatment of tuberculosis in Cambodia: a mixed-methods study. Infect Dis Poverty. 2020;9(1). doi:10.1186/s40249-020-00665-8

26. Angelo AT, Geltore TE, Asega T. Knowledge, attitude, and practices towards tuberculosis among clients visiting tepi general hospital outpatient departments, 2019. Infect Drug Resist. 2020;13:4559-4568. doi:10.2147/idr.S287288

27. Du L, Zhang Y, Lv X, et al. Prevalence of multidrug-resistant tuberculosis in Dalian, China: a Retrospective Study. Infect Drug Resist. 2021;14:1037-1047. doi:10.2147/idr.S294611

28. World Health Organization. Treatment of Tuberculosis Guidelines. World Health Organization; 2010.

29. Bello S, Afolabi RF, Ajayi DT, et al. Empirical evidence of delays in diagnosis and treatment of pulmonary tuberculosis: systematic review and meta-regression analysis. BMC Public Health. 2019:19820. doi:10.1186/s12889-019-7026-4.

30. Kim SH, Min J, Cho JY, et al. Clinical profiles and outcomes of pulmonary tuberculosis patients with delayed treatment at a tertiary hospital in South Korea. Ann Palliat Med. 2021;10(3):2948-2957. doi:10.21037/apm-20-1521

31. Yang Q, Tong YQ, Yin X, et al. Delays in care seeking, diagnosis and treatment of patients with pulmonary tuberculosis in Hubei, China. Int Health. 2020;12(2):101-106. doi:10.1093/inthealth/ihz036
32. Chandra A, Kumar R, Kant S, Krishnan A. Diagnostic pathways and delays in initiation of treatment among newly diagnosed tuberculosis patients in Ballabgarh, India. Am J Trop Med Hyg. 2021;104 (4):1321-1325. doi:10.4269/ajtmh.20-1297

33. Wang XL, He WC, Lei J, Liu GT, Huang F, Zhao YL. Impact of COVID-19 pandemic on pre-treatment delays, detection, and clinical characteristics of tuberculosis patients in Ningxia Hui Autonomous Region, China. Front Public Health. 2021;9644536. doi:10.3389/ fpubh.2021.644536

34. Haq K, McElhaney JE. Ageing and respiratory infections: the airway of ageing. Immunol Lett. 2014;162(1 Pt B):323-328. doi:10.1016/j. imlet.2014.06.009

35. Rajagopalan S. Tuberculosis in Older Adults. Clin Geriatr Med. 2016;32(3):479-491. doi:10.1016/j.cger.2016.02.006

36. Shu CC, Lee CH, Lee MC, Wang JY, Yu CJ, Lee LN. Hepatotoxicity due to first-line anti-tuberculosis drugs: a five-year experience in a Taiwan medical centre. Int $J$ Tuberc Lung Dis. 2013;17 (7):934-939. doi:10.5588/ijtld.12.0782

37. Lee CH, Wang JY, Lin HC, et al. Treatment delay and fatal outcomes of pulmonary tuberculosis in advanced age: a retrospective nationwide cohort study. BMC Infect Dis. 2017;17:11.449. doi:10.1186/ s12879-017-2554-y

38. Horton KC, MacPherson P, Houben R, White RG, Corbett EL. Sex differences in tuberculosis burden and notifications in low- and middle-income countries: a systematic review and meta-analysis. PLos Med. 2016;13(9):e1002119. doi:10.1371/journal.pmed.1002119

39. Dooley KE, Chaisson RE. Tuberculosis and diabetes mellitus: convergence of two epidemics. Lancet Infect Dis. 2009;9(12):737-746. doi:10.1016/s1473-3099(09)70282-8

40. Wang CH, Yu CT, Lin HC, Liu CY, Kuo HP. Hypodense alveolar macrophages in patients with diabetes mellitus and active pulmonary tuberculosis. Tuberc Lung Dis. 1999;79(4):235-242. doi:10.1054/ tuld.1998.0167

41. Getahun H, Matteelli A, Chaisson RE, Raviglione M. Latent Mycobacterium tuberculosis Infection. New Engl J Med. 2015;372 (22):2127-2135. doi:10.1056/NEJMra1405427

42. Nijland HMJ, Ruslami R, Stalenhoef JE, et al. Exposure to rifampicin is strongly reduced in patients with tuberculosis and type 2 diabetes. Clin Infect Dis. 2006;43(7):848-854. doi:10.1086/507543

43. Riccardi N, Villa S, Alagna R, et al. Advantages and challenges of tailored regimens for drug-resistant tuberculosis: a StopTB Italia look into the future. Infect Drug Resist. 2020;13:2795-2800. doi:10.2147/ idr.S257480
Infection and Drug Resistance

\section{Publish your work in this journal}

Infection and Drug Resistance is an international, peer-reviewed openaccess journal that focuses on the optimal treatment of infection (bacterial, fungal and viral) and the development and institution of preventive strategies to minimize the development and spread of resistance. The journal is specifically concerned with the epidemiology of antibiotic resistance and the mechanisms of resistance development and diffusion in both hospitals and the community. The manuscript management system is completely online and includes a very quick and fair peerreview system, which is all easy to use. Visit http://www.dovepress.com/ testimonials.php to read real quotes from published authors. 